\title{
Elucidation and quantification of the factors underlying bond-length variation in inorganic solids for the design of non-oxide materials with superior functional properties
}

\author{
O.C. Gagné ${ }^{1}$, F.C. Hawthorne ${ }^{2}$, R.M. Hazen ${ }^{1}$ \\ ${ }^{1}$ Geophysical Laboratory, Carnegie Institution for Science, 5251 Broad Branch Rd. NW, Washington D.C., 20015 USA; \\ ${ }^{2}$ Department of Geological Sciences, University of Manitoba, 125 Dysart Rd, Winnipeg, MB, Canada
}

ogagne@carnegiescience.edu

Growing interest in the design of functional materials with increasingly complex crystal structures calls for a more detailed understanding of structure-property relationships in inorganic solids. Whereby functional material properties are often linked to irregular bond distances, deciphering the causal mechanisms underlying bond-length variation, and the extent to which bond lengths vary in solids, has important implications in the design of new materials and the optimization of their functional properties.

Investigation of the relation between bond-length variation and the expression of functional material properties begins with systematization of chemical-bonding behavior via large-scale bond-length dispersion analysis. Completion of the largest bond-length dispersion analysis to date for inorganic solids (177,446 reliable bond lengths hand-picked from 9210 crystal-structure refinements for oxides [1]; 6,770 bond lengths from 720 crystal-structure refinements for nitrides [2]; 33,626 bond lengths from 1832 crystal-structure refinements for chalcogenides [3]) recently enabled straightforward identification of anomalous (i.e. irregular) bonding behavior for all ions of the periodic table observed bonding to $\mathrm{O}^{2-}, \mathrm{N}^{3-}$, and $\mathrm{S}^{2-} / \mathrm{Se}^{2-} / \mathrm{Te}^{2-}$. In addition to comprehensive description of bond-length variations in inorganic solids, the large amount of data on anomalous coordination environments provided by this undertaking allows (1) conclusive resolution of the causal mechanisms underlying bond-length variation in inorganic solids, and (2) quantification of the extent to which these causal mechanisms result in bond-length variation.

In a sample of 266 highly irregular coordination polyhedra covering 85 transition-metal ion configurations bonded to $\mathrm{O}^{2-}$, the most common cause of bond-length variation is observed to be non-local bond-topological asymmetry - a widely overlooked phenomenon whose associated bond-length variation results from asymmetric patterns of a priori bond valences - followed closely by the pseudo Jahn-Teller effect (PJTE). Two new indices, $\Delta_{\text {topol }}$ and $\Delta_{\text {eryst }}$, calculated on the basis of crystallographic site, are proposed to quantify bond-length variation arising from bond-topological and crystallographic mechanisms in extended solids; $\Delta_{\text {topol is defined }}$ as the mean weighted deviation between the bond valences of a given polyhedron and that of its regular variant with equal bond

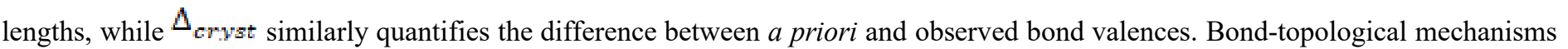
of bond-length variation are (1) non-local bond-topological asymmetry and (2) multiple-bond formation, while crystallographic mechanisms are (3) electronic effects (e.g. vibronic mixing, lone-pair stereoactivity), and (4) crystal-structure effects (e.g. structural incommensuration).

Comprehensive bond-length dispersion analyses for inorganic nitrides [2] and chalcogenides [3] reveal several "phenomenological gaps" compared to their oxide counterparts, thus providing synthetic opportunities via the transposing of anomalously bonded coordination units bearing functional properties into new compositional and/or structural spaces. Resolving the contribution of (static) bond-topological vs (tunable) crystallographic mechanisms of bond-length variation via the $\Delta_{\text {topol }}$ and $\Delta_{\text {eryst indices, combined with }}$ their spatial resolution within the coordination polyhedron and unit cell, is proposed to quantify the effective tunable extent of a functional property for a given crystal structure, e.g. via alteration of the responsible coordination unit(s). The known extent for which bond-topological and crystallographic mechanisms materialize into bond-length variations, provided by large-scale bond-length dispersion analyses, guides optimization of these properties within the constraints of physically realistic crystal structures. Such information is essential to the design of new materials with (1) increasingly complex crystal structures, and (2) superior functional properties.

[1] Gagné, O. C. \& Hawthorne, F. C. (2016). Acta Cryst. B72, 602-625; Gagné, O. C. (2018). Acta Cryst. B74, 49-62; Gagné, O. C. \& Hawthorne, F. C. (2018a). Acta Cryst. B74, 63-78; Gagné, O. C. \& Hawthorne, F. C. (2018b). Acta Cryst. B74, 79-96; Gagné, O. C. \& Hawthorne, F. C. ChemRxiv 11605698.

[2] Gagné, O. C. (2020). ChemRxiv. 11626974

[3] Gagné, O. C. et al. (2020). In preparation

Keywords: Bond-length dispersion analysis; bond-length variation; bond-topological asymmetry; materials design 